\title{
Normkonkretisierende Verwaltungsvorschriften im Umweltrecht: Normkonkretisierung als Normersetzung?
}

\section{Problemstellung}

Mit der sog. Wyhl-Entscheidung des Bundesverwaltungsgerichts ${ }^{1}$ wurde der Begriff der unmittelbar nach außen wirkenden und ggf. bindenden ,normkonkretisierenden Verwaltungsvorschriften" entwickelt, die seither als neubestimmte Normkategorie im Umweltrecht eine nicht zu unterschätzende Rolle gespielt haben. ${ }^{2}$ Ihre Notwendigkeit ergibt sich daraus, dass die zahlreichen unbestimmten Rechtsbegriffe im Umweltrecht konkretisierungsbedürftig sind. Demzufolge enthalten diese unbestimmten Rechtsbegriffe Beurteilungsermächtigungen für die zuständige Behörde, die daher zum Erlaß von normkonkretisierenden Verwaltungsvorschriften befugt ist. Nach neuerer Ansicht stützt sich die Legitimation der normkonkretisierenden Verwaltungsvorschriften sogar nicht nur auf die Ermächtigung der Legislative, sondern darüber hinaus auf die originäre Rechtsetzungskompetenz der Exekutive. Auch gerade deswegen stellen die normkonkretisierenden Verwaltungsvorschriften unter bestimmten Voraussetzungen nicht nur die unmittelbare Außenwirkung, sondern ferner eine verstärkte Bindungsintensität für die Verwaltungsgerichte dar. Allerdings bleibt dabei stets umstritten, weshalb und inwiefern diese normkonkretisierenden Verwaltungsvorschriften zur Begrenzung der gerichtlichen Kontrolldichte führen dürften. In Schriftum und Rechtsprechung wird die Meinung vertreten, dass die Verbindlichkeit der normkonkretisierenden Verwaltungsvorschriften aus den behördlichen Beurteilungs- bzw. Standardisierungsspielräumen, aus dem behördlichen technischen Sachverstand sowie aus der Komplexität und Dynamik der geregelten Materie erfolgt. Dementsprechend fungieren vornehmlich nicht die ermächtigenden unbestimmten Rechtsbegriffe, sondern vielmehr die technisch-wissenschaftlichen Erkenntnisfortschritte als Kontrollmaßstäbe für die (begrenzte) gerichtliche Überprüfung. Fraglich ist jedoch, ob diese Gründe, die übereinstimmend für die Eigenständigkeit und „funktionelle Richtigkeit“ der Exekutive sprechen, verfassungs- und verwaltungsrechtlich begründet sind. Nach Auffassung der vorliegenden Arbeit stellt im Umweltrecht die Begriffsentwicklung der normkonkretisierenden Verwaltungsvorschriften eine Missdeutung der umweltrechtlichen unbestimmten Rechtsbegriffe dar, sofern diese als unvollständige und daher bindungsunfähige Gesetzesnormen verstanden werden. Im Folgenden wird gezeigt, wie diese Grundannahme zur Entstehung der normkonkretisierenden Verwaltungsvorschriften und weiterhin zu deren Bindungswirkung für die Gerichte beigetragen hat. Dadurch soll verdeutlicht werden, dass die (angebliche) Reduzierung der gerichtlichen Kontrolldichte bei den normkonkretisierenden Verwaltungsvorschriften die Gesetzesbindung der unbestimmten Rechtsbegriffe auflösen und damit die gerichtliche Kontrollfunktion beeinträchtigen würde.

1 BVerwGE 72, 300 (320).

2 Zur gerichtlichen Anerkennung der Außen- und Bindungswirkung der normkonkretisierenden Verwaltungsvorschriften vgl. neuerdings auch BVerwGE 129, 209 (211). 


\section{Normkonkretisierende Verwaltungsvorschriften als Ausfüllung von unbestimmten Rechtsbegriffen in Anerkennung behördlicher Beurteilungsspielräume}

Die Begriffsentwicklung der normkonkretisierenden Verwaltungsvorschriften durch die Wyhl-Entscheidung des Bundesverwaltungsgerichts lässt sich auf die Eigenschaften des Umweltrechts zurückführen, welches sich durch die Abhängigkeit von naturwissenschaftlich-technischem Sachverstand ${ }^{3}$ und heutzutage ferner durch hohe Dynamik und starke Ungewissheit infolge der Europäisierung und Internationalisierung ${ }^{4}$ auszeichnet. Daher wird im Allgemeinen angenommen, dass allein der Gesetzgeber bzw. das parlamentarische Gesetz nicht in der Lage ist, im Bereich des Umweltrechts umfassende oder sogar nur wesentliche Regelungen vorzunehmen, so dass die im Umweltrecht vielfach verwendeten unbestimmten Rechtsbegriffe wie „Stand von Wissenschaft und Technik“ und „schädliche Umwelteinwirkungen“ ohne weitere Konkretisierungen nicht steuerungsfähig sein könnten. ${ }^{5}$ Vor diesem Hintergrund liegt es nahe, dass die unbestimmten Rechtsbegriffe des Umweltrechts häufig nicht, wie im Rahmen des traditionellen Ordnungsverwaltungsrechts, auf eine umfassende Verwaltungsgerichtsbarkeit, ${ }^{6}$ sondern vielmehr auf die behördlichen Beurteilungsspielräume verweisen: ${ }^{7} \mathrm{Da}$

3 Vgl. dazu etwa $R$. Wahl, Risikobewertung der Exekutive und richterliche Kontrolldichte Auswirkungen auf das Verwaltungs- und das gerichtliche Verfahren, NVwZ 1991, S. 409 ff., 410 ; R. Breuer, Gerichtliche Kontrolle der Technik. Gegenpol zu privaten Option und administrativer Standardisierung, NVwZ 1988, S. 104 ff., 111 f.; M. Schmidt-Preuß, Das Atomrecht als Referenzgebiet des Verwaltungsrechts, DVB1. 2000, S. 767 ff., 767; K.-H. Ladeur, Das Umweltrecht der Wissensgesellschaft: Von der Gefahrenabwehr zum Risikomanagement, 1995 , S. $79 \mathrm{ff}$.

$4 \mathrm{Zu}$ Dimensionen der Europäisierung und Internationalisierung des Umweltrechts vgl. nur $W$. Durner, Internationales Umweltverwaltungsrecht, in: C. Möllers/A. Voßkuhle/C. Walter (Hrsg.), Internationales Verwaltungsrecht, 2007, S. 121 ff., 142 ff.; M. Rossi, Europäisiertes internationales Umweltverwaltungsrecht, in: Möllers/Voßkuhle/Walter (Hrsg.), ebenda, S. $165 \mathrm{ff}$.

5 Vgl. z. B. T. v. Danwitz, Normkonkretisierende Verwaltungsvorschriften und Gemeinschaftsrecht, VerwArch 84 (1993), S. 73 ff., 93; R. Breuer, Tendenzwende des Rechtsschutzes? Betrachtungen zu den $\S \S 43 \mathrm{ff}$. UGB-KomE, in: UTR 1998, S. $161 \mathrm{ff} ., 172$.

6 Zur klassischen Annahme, die behördliche Auslegung und Anwendung unbestimmter Rechtsbegriffe unterliege einer uneingeschränkten verwaltungsgerichtlichen Überprüfung, vgl. nur D. Jesch, Unbestimmter Rechtsbegriff und Ermessen in rechtstheoretischer und verfassungsrechtlicher Sicht, AöR 82 (1957), S. 163 ff., 212, 236 ff.; C. H. Ule, Zur Anwendung unbestimmter Rechtsbegriffe im Verwaltungsrecht, in: Gedächtnisschrift für W. Jellinek, 1955, S. $309 \mathrm{ff} ., 314 \mathrm{ff}$.

7 Vgl. dazu H. Schulze-Fielitz, Neue Kriterien für die verwaltungsgerichtliche Kontrolldichte bei der Anwendung unbestimmter Rechtsbegriffe, JZ 1993, S. 772 ff., 774; U. Di Fabio, Verwaltungsvorschriften als ausgeübte Beurteilungsermächtigung: Plädoyer für eine Neubestimmung der normkonkretisierenden Verwaltungsvorschriften im System der Rechtsquellen, DVBl. 1992, S. 1338 ff., 1338; K.-H. Ladeur, Normkonkretisierende Verwaltungsvorschriften als Recht privat-öffentlicher Kooperationsverhältnisse - Das Beispiel der gemeinsamen Richtlinien der Landesmedienanstalten und die Perspektiven des Verwaltungsrechts der Informationsgesellschaft, DÖV 2000, S. 217 ff., 221 f.; E. Pache, Tatbestandliche Abwägung und Beurteilungsspielraum: Zur Einheitlichung administrativer Entscheidungsfreiräume und zu deren Konsequenzen im verwaltungsgerichtlichen Verfahren - Versuch einer Modernisierung, 2001, S. $80 \mathrm{f}$. 
der Gesetzgeber nur inhaltsknappe Regelungen bzw. ,unbestimmte“ Rechtsbegriffe erlassen kann, muss die zuständige Behörde zur Anwendung dieser unbestimmten Rechtsbegriffe die Konkretisierungsaufgabe übernehmen. In diesem Zusammenhang lässt sich verstehen, weshalb die Eigenständigkeit der Verwaltung gegenüber dem Gesetz im Umweltrecht mit Nachdruck hervorgehoben wird. ${ }^{8}$ Besonders im Rahmen des Kernbegriffs „Risikovorsorge“ betont das Bundesverwaltungsgericht, die Verantwortung für die Risikoermittlung und -bewertung trage die Exekutive. ${ }^{9}$ Demzufolge ergibt sich die behördliche Kompetenz zur Konkretisierung der umweltrechtlichen unbestimmten Rechtsbegriffe nicht nur aus der ausdrücklichen Ermächtigung der unbestimmten Rechtsbegriffe, sondern vielmehr daraus, dass die Exekutive über funktionelle und besondere fachliche Legitimation verfügt.

Gerade im Hinblick auf die wesentliche Rolle der Exekutive wurden die nach außen wirkenden normkonkretisierenden Verwaltungsvorschriften entwickelt. Grundlage dafür ist zuallererst der der Verwaltung eingeräumte Beurteilungsspielraum, welcher sich aus der Unvollkommenheit der unbestimmten Rechtsbegriffe ergibt. ${ }^{10}$ Innerhalb des Beurteilungsspielraums also ist die Behörde dazu befugt, Verwaltungsvorschriften zu erlassen, um die unvollkommenen unbestimmten Rechtsbegriffe auszufüllen. Wie das Bundesverwaltungsgericht darlegt: „Diese Verwaltungsvorschriften dienen nämlich der Ausfüllung eines der Verwaltung eingeräumten Beurteilungsspielraums. Mit ihnen wird die Ausübung dieses Beurteilungsspielraums von der Einzelentscheidung im jeweiligen Verwaltungsakt in eine abstrakt generalisierende Regelung vorverlagert, um so die Einheitlichkeit des Verwaltungshandelns sicherzustellen." 11 Auf dieser Erkenntnisgrundlage wendet sich das Bundesverwaltungsgericht in der Whyl-Entscheidung davon ab, die die unbestimmten Rechtsbegriffe ausfüllenden Verwaltungsvorschriften als „antizipiertes Sachverständigengutachten"12 anzusehen. Vielmehr hätten diese Verwaltungsvorschriften eine normkonkretisierende Funktion und seien ,im Gegensatz zu lediglich norminterpretierenden Verwaltungsvorschriften für die Verwaltungsgerichte innerhalb der von der Norm gesetzten Grenzen verbindlich“. ${ }^{13}$ Insofern zeigt sich, dass die Verbindlichkeit dieser Verwaltungsvorschriften, die in der Regel nur Innenwirkung entfalten könnten, sich nicht nur nach außen manifestiert, sondern insbesondere gegenüber dem Verwaltungsgericht wirkt. Demzufolge sei die „normkonkretisierende Verwal-

8 Vgl. etwa Schulze-Fielitz (Fn. 7), S. 779; W. Hoffmann-Riem, Verwaltungsrechtsreform Ansätze am Beispiel des Umweltrechts, in: W. Hoffmann-Riem/E. Schmidt-Aßmann/G. F. Schuppert (Hrsg.), Reform des allgemeinen Verwaltungsrechts: Grundfragen, 1993, S. 115 ff., 130 ff.; M. Kloepfer, Umweltschutzrecht, 2008, S. 46.

9 BVerwGE 72, 300 (316 f. unter Berufung auf BVerfGE 49, 89 (140)). Vgl. dort ferner S. 315: „Die Struktur des in $\S 7$ Abs. 2 Nr. 3 AtG verwendeten Vorsorgebegriffs läßt es nicht zu, ihn im herkömmlichen Sinne als unbestimmten Rechtsbegriff ohne Beurteilungsspielraum zu bezeichnen."

10 Vgl. nur Schulze-Fielitz (Fn. 7), S. 780; H. Maurer, Allgemeines Verwaltungsrecht, 17. Aufl., 2009, S. 632; R. Breuer, Umweltschutzrecht, in: E. Schmidt-Aßmann/F. Schoch (Hrsg.), Besonderes Verwaltungsrecht6, 14. Aufl. 2008, § 5, Rn. 181; Kloepfer (Fn. 8), S. 49.

11 BVerwGE 107, 338 (341).

12 So BVerwGE 55, 250 (256) unter Berufung auf R. Breuer, Die rechtliche Bedeutung der Verwaltungsvorschriften nach $\S 48$ BImSchG im Genehmigungsverfahren, DVB1. 1978, S. 28 ff., 35 .

13 BVerwGE 72, 300 (320). 
tungsvorschrift" vom Verwaltungsgericht nur daraufhin zu überprüfen, ob sie auf willkürfreien Ermittlungen beruhe und in der Lage sei, die Einzelentscheidung der Behörde zu hinreichend konservativen Abschätzungen zu führen. ${ }^{14}$ Zur Voraussetzung für die Außen- und Bindungswirkung der normkonkretisierenden Verwaltungsvorschriften hat das Bundesverwaltungsgericht später noch hinzugefügt: „Normkonkretisierende Wirkung kann nämlich einer Verwaltungsvorschrift nur dann zukommen, wenn die Exekutive bei ihrem Erlaß höherrangigen Geboten und dem für deren Konkretisierung wesentlichen Erkenntnis- und Erfahrungsstand Rechnung getragen hat. Auch darf die Verwaltungsvorschrift nicht durch Erkenntnisfortschritte in Wissenschaft und Technik überholt sein. Schließlich kann einer Verwaltungsvorschrift nur dann ausnahmsweise normkonkretisierende Wirkung zukommen, wenn dem Erlaß ein umfangreiches Beteiligungsverfahren vorangeht, dessen Zweck es ist, vorhandende Erfahrungen und den Stand der wissenschaftlichen Erkenntnis auszuschöpfen. Nur dann verkörpert sie nämlich in hohem Maße wissenschaftlich-technischen Sachverstand."15 Diese Ausführungen halten zwar daran fest, dass auch innerhalb des behördlichen Beurteilungsspielraums die (normkonkretisierenden) Verwaltungsvorschriften der verwaltungsgerichtlichen Kontrolle unterliegen. Gleichzeitig stellen sie aber klar, dass hier die verwaltungsgerichtliche Überprüfung keineswegs uneingeschränkt sein darf. ${ }^{16}$ Besonders bemerkenswert ist dabei, dass im Rahmen der Verwaltungsgerichtsbarkeit die konkretisierten unbestimmten Rechtsbegriffe überwiegend als Ermächtigungs-, nicht aber als Bindungsquelle angesehen werden. Dies geht, wie angedeutet, wiederum auf die Eigenständigkeit der Verwaltung im Umweltrecht zurück.

\section{Die Debatte um die Bindungswirkung der normkonkretisierenden} Verwaltungsvorschriften

\section{Die Rezeption der normativen Ermächtigungslehre}

Während die unmittelbare Wirkung der normkonkretisierenden Verwaltungsvorschriften nach außen, besonders gegenüber dem Verwaltungsgericht, seit einiger Zeit anerkannt worden ist, ist ihre Rechtfertigungsgrundlage nach wie vor umstritten. Nach Ansicht derjenigen, die an der sog. normativen Ermächtigungslehre festhalten, dürfen die normkonkretisierenden Verwaltungsvorschriften nur dann ihre unmittelbare Außenwirkung entfalten, wenn sie aus der gesetzlichen Ermächtigung zur abstrakt-generellen Normkonkretisierung und ferner aus der gesetzlichen Ausgestaltung des administrativen Normsetzungsverfahrens folgen. ${ }^{17}$ Anders ausgedrückt stützt sich in Anlehnung an die

14 BVerwGE 72, 300 (321).

15 BVerwGE 107, 338 (341 f.). Vgl. ferner BVerwGE 110, 216 (219); 114, 342 (455 f.).

16 Vgl. in diesem Zusammenhang auch OVG Lüneburg, DVBl. 1985, S. 1322 ff., 1323.

17 So z. B. E. Schmidt-Aßmann, in: Maunz/Dürig, Grundgesetz-Kommentar, Bd. III, 2007, Art. 19 Abs. 4, Rn. 206 a; E. Schmidt-Aßmann, Das allgemeine Verwaltungsrecht als Ordnungsidee: Grundlagen und Aufgaben der verwaltungsrechtlichen Systembildung, 2. Aufl., 2006, S. 330; E. Rehbinder, Ziele, Grundsätze, Strategien und Instrumente des Umweltschutzes, in: K. Hansmann/D. Sellner (Hrsg.), Grundzüge des Umweltrechts, 3. Aufl., 2007, S. 123 ff., 277 f. Vgl. auch Di Fabio (Fn. 7), S. 1344 f. 
normative Ermächtigungslehre die unmittelbare Außenwirkung der normkonkretisierenden Verwaltungsvorschriften auf die gesetzliche Ermächtigung, die eine letztverbindliche Standardisierungsbefugnis der Exekutive begründet. ${ }^{18}$ Demzufolge soll die Bindungsintensität der normkonkretisierenden Verwaltungsvorschriften davon abhängen, ob ihre Konkretisierung innerhalb des gesetzlichen Rahmens erfolgt. ${ }^{19}$ Da aber der gesetzliche Rahmen bzw. der konkretisierungsbedürftige unbestimmte Rechtsbegriff meist für überwiegend inhaltsknapp gehalten wird, ${ }^{20}$ kann sich die (beschränkte) verwaltungsgerichtliche Überprüfung nur an die anderen Kontrollmaßstäbe wenden, nämlich ob die normkonkretisierenden Verwaltungsvorschriften durch Erkenntnisfortschritte in Wissenschaft und Technik überholt sind und ob atypische Einzelfälle vorliegen. ${ }^{21}$ Nach Auffassung der Anhänger der normativen Ermächtigungslehre erregt also die Bindungswirkung der normkonkretisierenden Verwaltungsvorschriften für die Gerichte deshalb keine verfassungsrechtliche Bedenken, weil sie sich einerseits aus der gesetzlichen Ermächtigung ergibt und andererseits immerhin mit der Wächterfunktion der Gerichte verbunden ist, deren Konzentration auf ,zwischenzeitliche Erkenntnisfortschritte“ oder ,atypische Einzelfälle“ gerade die Eigenart bzw. die „labile und gegenüber Erkenntnisfortschritten offenere Wirkung" der normkonkretisierenden Verwaltungsvorschriften verkörpert, ,die sich in einer Regelvermutung ausdrückt, im atypischen Fall oder durch Entwicklungen aber ausgeräumt werden kann“. ${ }^{22}$

\section{Die Anerkennung der originären Rechtsetzungsbefugnis der Exekutive}

Demgegenüber gehen diejenigen, die die Eigenständigkeit der Verwaltung im Gefüge der Staatsfunktionen herausstellen, davon aus, daß die unmittelbare Außenwirkung der normkonkretisierenden Verwaltungsvorschriften auf der administrativen originären Rechtsetzungskompetenz beruht. Diese Annahme setzt voraus, dass Art. 80 Abs. 1 GG nur dem Vorbehaltsbereich der Legislative zugehört und daher das außenwirkende Administrativrecht nicht ausschließt. Demzufolge sind Verwaltungsvorschriften im Funk-

$18 \mathrm{Ob}$ und inwiefern dabei gesetzliche Standardisierungs- und Beurteilungsermächtigungen zu unterscheiden sind, steht auf einem anderen Blatt. Vgl. dazu näher J. Saurer, Die neueren Theorien zur Normkategorie der Verwaltungsvorschriften, VerwArch 97 (2006), S. 249 ff., 256 f.; Pache (Fn. 7), S. 104 ff.; Breuer (Fn. 5), S. 174-176.

19 Zur gerichtlichen Überprüfung der Recht- und Gesetzmäßigkeit der normkonkretisierenden Verwaltungsvorschriften vgl. H. Jarass, Bindungswirkung von Verwaltungsvorschriften, JuS 1999, S. 105 ff., 110; BVerwG, NVwZ 1988, S. 824 ff., 825.

20 Vgl. z. B. Jarass (Fn. 19), S. 110.

21 Vgl. dazu E. Schmidt-Aßmann, in: Maunz/Dürig, Grundgesetz-Kommentar, Bd. III, 2007, Art. 19 Abs. 4, Rn. 206 b; Breuer (Fn. 10), Rn. 181; Jarass (Fn. 19), S. 110 f.; Pache (Fn. 7), S. $95 \mathrm{f}$.

22 Schmidt-Aßmann, Das allgemeine Verwaltungsrecht als Ordnungsidee (Fn. 17), S. 329. 
tionsbereich der Exekutive angesiedelt. ${ }^{23}$ „Es handelt sich um administratives Vollzugsrecht, das dazu dient, den Vollzug zu ermöglichen und zu erleichtern. Mit ihrem Erlaß übt die Verwaltung eine eigene Funktion aus." 24 So gesehen unterscheidet sich die normkonkretisierende Verwaltungsvorschrift von der Rechtsverordnung vornehmlich dadurch, dass sie nicht mehr mit dem Gesetz bzw. der gesetzlichen Ermächtigung streng verknüpft ist: „Während bei der Rechtsverordnung eine Bindung durch ein bestimmtes Gesetzesprogramm besteht, stützt sich die Verwaltung beim Erlaß von Verwaltungsvorschriften auf den Auftrag zur rechtsdisziplinierten Erfüllung der gesetzlich begründeten Verwaltungsaufgabe; sie ist insofern bei dieser Vollzugsaufgabe nur thematisch gebunden. “25 Nach diesem ,verwaltungsfreundlichen“ Ansatz also gründet die normkonkretisierende Verwaltungsvorschrift einerseits auf dem Gesetzesauftrag, sie unterliegt andererseits aber keiner strengen Gesetzesbindung, denn sie ist gerade dazu berufen, das unvollständige Gesetz nicht nur auszulegen, sondern auch auszugestalten. ${ }^{26}$ Genau mit diesem Charakter entfaltet die normkonkretisierende Verwaltungsvorschrift ihre Bindungswirkung für die Gerichte. Zwar bleibt dabei gerichtlich noch zu prüfen, ob die Verwaltungsvorschrift bei atypischen Sachverhalten oder lokalen Besonderheiten nicht anwenbar ist, ob sie auf willkürfreien Ermittlungen und Bewertungen beruht und ob sie nicht durch gesicherte neue Erkenntnisse überholt ist. ${ }^{27}$ Die Verbindlichkeit der normkonkretisierenden Verwaltungsvorschriften gegenüber dem Verwaltungsgericht verdeutlicht sich in Anerkennung der originären Rechtsetzungsbefugnis der Exekutive aber inbesondere dadurch, dass in erster Linie nicht die gerichtliche Wächterfunktion, sondern vielmehr die ,politische und demokratische Kontrolle und Sanktionsunterworfenheit" der Verwaltungsvorschriften ${ }^{28}$ in den Vordergrund gerückt wird. Vor allem unter Beachtung ,funktionell-rechtlicher Gesichtspunkte“ ${ }^{\text {" } 9}$ legt dieser Ansatz sogar ausdrücklich dar, dass die Kontrolle der Verwaltungsgerichte gegenüber

23 In diese Richtung schon W. Krebs, Zur Rechtsetzung der Exekutive durch Verwaltungsvorschriften, VerwArch 70 (1979), S. 259 ff., 265 ff., 269ff. Vgl. auch A. Leisner, Verwaltungsgesetzgebung durch Erlasse, JZ 2002, S. 219 ff., 226 f., 230; F. Ossenbühl, Rechtsquellen und Rechtsbindungen der Verwaltung, in: H.-U. Erichsen/D. Ehlers (Hrsg.), Allgemeines Verwaltungsrecht, 12. Aufl., 2002, §6, Rn. 42; F. Ossenbühl, Autonome Rechtsetzung der Verwaltung, in: J. Isensee/P. Kirchhof (Hrsg.), HStR , 2. Aufl., 1996, § 65, Rn. 65; H.-D. Horn, Die grundrechtsunmittelbare Verwaltung. Zur Dogmatik des Verhältnisses zwischen Gesetz, Verwaltung und Individum unter dem Grundgesetz, 1999, S. 64 ff. Dazu kritisch M. Jachmann, Die Bindungswirkung normkonkretisierender Verwaltungsvorschriften. Anmerkungen zu einer Rechtsetzungsfunktion der rechtsanwendenden Verwaltung aus methodologischer sowie verfassungsrechtlicher Sicht, Die Verwaltung 1995, S. 17 ff., 23 ff.; Saurer (Fn. 18), S. 266 f.

24 H. Hill, Normkonkretisierende Verwaltungsvorschriften, NVwZ 1989, S. 401 ff., 405.

25 Hill (Fn. 24), S. 405. Vgl. dort ferner S. 406: „Stellten sich Rechtsverordnungen als nachfolgender, abgespaltener Teil eines Gesetzes dar, so kommt normkonkretisierenden Verwaltungsvorschriften der Charakter eines vorweggenommenen Teils der Verwaltungsentscheidung zu."

26 Vgl. in diesem Zusammenhang auch Di Fabio (Fn. 17), S. 1340: „Es geht deshalb nicht eigentlich um Gesetzesauslegung im juristisch-methodischen Sinne, sondern um untergesetzliche Normausgestaltung."

27 Vgl. Hill (Fn. 24), S. 409 ff.

28 So Hill (Fn. 24), S. $408 \mathrm{f}$.

29 Vgl. in diesem Zusammenhang auch v. Danwitz (Fn. 5), S. 92 f. 
staatsleitenden Regierungsakten tendenziell den Charakter der Kontrolle der Verfassungsgerichte gegenüber Parlamentsakten annehme: „Das bedeutet Respektierung eines Gestaltungsspielraums der Bundesregierung wegen ihrer politisch-demokratischen Verantwortung und Sanktionsunterworfenheit."30

\section{Zwischenergebnis}

Dem Vorhergehenden lässt sich entnehmen, dass nach den beiden erwähnten Ansätzen zur unmittelbaren Außenwirkung der normkonkretisierenden Verwaltungsvorschriften nicht mehr die ermächtigenden unbestimmten Rechtsbegriffe, sondern entweder die rechtsstaatlich allgemeingültigen Richtlinien oder sogar andere Faktoren, wie etwa die technisch-wissenschaftlichen Erkenntnisfortschritte, maßgebend für die gerichtliche Kontrolle sind, die infolge der Bindungswirkung der normkonkretisierenden Verwaltungsvorschriften gewissermaßen begrenzt ist. Der erstere Ansatz sieht die gesetzliche Ermächtigung zwar als Rechtfertigungsgrundlage für die Verbindlichkeit der normkonkretisierenden Verwaltungsvorschriften an. Dennoch wird dabei nicht, wie im Rahmen der Rechtsverordnung bzw. des Art. 80 Abs. 1 GG, die bindende Bedeutung, sondern vielmehr die kompetenzübertragende Rolle der gesetzlichen Ermächtigung herausgestellt. Auf dieser Grundlage überrascht es nicht, dass hier die ermächtigenden unbestimmten Rechtsbegriffe nicht zur Einschränkung der Bindungswirkung normkonkretisierender Verwaltungsvorschriften für die Gerichte dienen könnten - diese sei vornehmlich durch die Aktualisierung technisch-wissenschaftlicher Erkenntnisse zu schwächen und bleibe gerade deswegen unbedenklich. Der letztere Ansatz geht ein Stück weiter, indem die originäre Rechtsetzungskompetenz der Exekutive aus funktionell-rechtlichen Gesichtspunkten hervorgehoben und die normkonkretisierende Verwaltungsvorschrift als ,eigener Funktionsbereich“ der Verwaltung anerkannt wird. Daher liegt es nahe, dass die normkonkretisierende Verwaltungsvorschrift gerade die ,administrative Gesetzgebung“" verkörpere, wobei weder der ermächtigende unbestimmte Rechtsbegriff noch die gerichtliche Überprüfung, sondern die funktionell-rechtliche Legitimation bzw. Sachverstand, Flexibilität und Dynamik der Verwaltung im Mittelpunkt stehe. Dementsprechend hänge die Verbindlichkeit der normkonkretisierenden Verwaltungsvorschriften gegenüber dem Gericht auch nicht von der gesetzlichen Ermächtigung ab. Sondern sie werde besonders dadurch begrenzt, dass die normkonkretisierenden Verwaltungsvorschriften durch technisch-wissenschaftliche Erkenntnisfortschritte überholt sein könnten. Nach den beiden Ansätzen also führt die normkonkretisierende Verwaltungsvorschrift deshalb zur Rücknahme der verwaltungsgerichtlichen Kontrolldichte, weil sie substantiell nicht mehr mit dem ermächtigenden unbestimmten Rechtsbegriff, sondern, nach allgemeinem Eindruck, immer wieder mit dem naturwis-

30 Hill (Fn. 24), S. 409. 
senschaftlich-technischen Sachverstand verbunden ist. ${ }^{31}$ Allerdings bestätigt die verstärkte Außen- und Bindungswirkung der normkonkretisierenden Verwaltungsvorschriften nicht deren Annährung an die Rechtsverordnungen. Im Gegenteil: Die beiden erwähnten Gesichtspunkte sind davon ausgegangen, dass die normkonkretisierenden Verwaltungsvorschriften von den Rechtsverordnungen zu unterscheiden sind, denn diese sind im Rahmen des Art. 80 Abs. 1 GG streng an die ermächtigenden Gesetze gebunden. Daher ist nach den Anhängern der normativen Ermächtigungslehre die normkonkretisierende Verwaltungsvorschrift immer wieder auf die behördlichen Beurteilungsspielräume zurückzuführen, während die Vertreter der administrativen originären Rechtsetzungsbefugnis die normkonkretisierende Verwaltungsvorschrift sogar gewissermaßen als substantiellen Bestandteil des (parlamentarischen) Gesetzes einstufen, ${ }^{32}$ indem dieser gerade die Funktion zukommt, eine ,wesentliche Vorabentscheidung“ zu treffen und dergestalt die Verwaltungsentscheidung ,rechtsstaatlich und grundrechtlich vorhersehbar zu machen". 33

\section{$I V$. Die Begriffsentwicklung der normkonkretisierenden Verwaltungsvorschriften aus dem Missverständnis der unbestimmten Rechtsbegriffe im Rahmen des Umweltrechts}

Die vorstehenden Ausführungen haben verdeutlicht, daß die normkonkretisierenden Verwaltungsvorschriften insgesamt zur Sicherung eines eigenständigen, d. h. gerichtlicher Kontrolle nur beschränkt zugänglichen administrativen Entscheidungsfreiraums entwickelt wurden. ${ }^{34}$ Wie oben dargelegt, setzt diese Zielbestimmung die Unvollständigkeit und Konkretisierungsbedürftigkeit der umweltrechtlichen unbestimmten Rechtsbegriffe voraus. Genau auf dieser Grundlage ist die ausgestaltende Funktion der normkonkretisierenden Verwaltungsvorschriften weitgehend anerkannt, die zu einer begrenzten verwaltungsgerichtlichen Kontrolle führen sollen. Auffallend ist aber zugleich, dass nach herrschender Auffassung selbst die Bindungsintensität der normkonkretisierenden Verwaltungsvorschriften gegenüber dem Gericht von mehreren Faktoren abhängen muss. Vor allem dann, wenn die normkonkretisierende Verwaltungsvorschrift den aktualisierten technisch-wissenschaftlichen Erkenntnissen nicht mehr entspreche, oder wenn aufgrund dieser Erkenntnisse die normkonkretisierende Verwaltungsvor-

31 Vgl. in diesem Zusammenhang auch Schmidt-Preuß (Fn. 3), S. 776: „Hat das Verwaltungsgericht Zweifel an der Sachrichtigkeit des Regelwerks, weil dieses auf falschen Angaben beruht, durch neue Erkenntnisse überholt ist oder atypischen Einzelfälle nicht hinreichend Rechnung trägt, weist es die exekutivische Auslegungsofferte zurück. Da die Verwaltungsvorschriften aus institutionalisiertem Sachverstand erwachsen, kann man davon ausgehen, dass hierzu nur in Ausnahmefällen Anlass bestehen wird." (Hervorhebung im Original)

32 In diesem Zusammenhang kritisch auch J. Wolf, Die Kompetenz der Verwaltung zur „Normsetzung" durch Verwaltungsvorschriften, DÖV 1992, S. 849 ff., 856.

33 So wiederum Hill (Fn. 24), S. 407. Vgl. in diesem Zusammenhang auch I. Lamb, Kooperative Gesetzeskonkretisierung: Verfahren zur Erarbeitung von Umwelt- und Technikstandards, 1995, S. 41; Breuer (Fn. 5), S. 172. Der „Gesetzescharakter“ der normkonkretisierenden Verwaltungsvorschriften zeigt sich auch durch deren politischen Bewertungskomponente, welche bereits die Wyhl-Entscheidung hervorgehoben hat.

34 Vgl. dazu nur R. Hendler, Umweltrechtliche Grenzwerte in der Gerichts- und Verwaltungspraxis, DÖV 1998, S. 481 ff., 489. 
schrift bei atypischen Sachverhalten nicht anwendbar sei, solle sie ihre Bindungswirkung verlieren. Insofern lässt sich sagen, dass die erhebliche Komplexität und Dynamik der zu regelnden Materie bzw. der besondere Sachverstand der Verwaltung die Verbindlichkeit der normkonkretisierenden Verwaltungsvorschriften gegenüber dem Gericht einerseits begründet, andererseits aber auch beschränkt. In dieser Hinsicht ist dem EuGH insoweit zuzustimmen, als er davon ausging, die allgemeinverbindliche Außenwirkung von (normkonkretisierenden) Verwaltungsvorschriften sei in der deutschen verwaltungsrechtlichen Lehre nach wie vor umstritten. ${ }^{35}$ Abgesehen von der Frage, ob die normkonkretisierende Verwaltungsvorschrift geeignet ist, EU-Richtlinien in das deutsche Recht umzusetzen, ${ }^{36}$ hat die schwankende Stellung dieser „Normkategorie“ zur Folge, dass ihre Einführung in die Verwaltungsrechtsdogmatik nicht nur die Kompetenzabgrenzung von Legislative und Exekutive erschwert, sondern darüber hinaus die Probleme der Aufgabenverteilung von Exekutive und Judikative verschäft.

Die Begriffsentwicklung der normkonkretisierenden Verwaltungsvorschriften stützt sich auf die Grundannahme, die im Umweltrecht vielfach verwendeten unbestimmten Rechtsbegriffe wie „Stand von Wissenschaft und Technik“ und „schädliche Umwelteinwirkungen"seien für die Rechtsanwendung inhaltsleer und daher maßstablos. Bereits dieser Ausgangspunkt verkennt die Eigenschaft dieser unbestimmten Rechtsbegriffe im Rahmen des Umweltrechts. Er setzt die klassische, vor allem im traditionellen Ordnungsverwaltungsrecht weit verbreitete Vorstellung voraus, dass das (parlamentarische) Gesetz dem Bestandsschutz bzw. dem Wirksamwerden eines stabilisierenden und retardierenden Moments gegenüber der Dynamik politischen und sozialen Lebens dienen solle, ${ }^{37}$ während die Verwaltung im Gegenteil durch ihren dynamischen Charakter gekennzeichnet sei. ${ }^{38}$ Erst mit dieser Voraussetzung ist die herrschende Meinung davon überzeugt, die inhaltliche Offenheit der zahlreichen umweltrechtlichen unbestimmten

35 Vgl. EuGH, NVwZ 1991, 866, 868.

$36 \mathrm{Zu}$ dieser Debatte vgl. statt vieler R. Steiling, Mangelnde Umsetzung von EG-Richtlinien durch den Erlaß und die Anwendung der TA Luft, NVwZ 1992, S. 134 ff.; Wolf (Fn. 32), S. 858 ff.; v. Danwitz (Fn. 5), S. 80 ff.; F. Ossenbühl, 40 Jahre Bundesverwaltungsgericht: Bewahrung und Fortentwicklung des Rechtsstaates, DVBl. 1993, S. 753 ff., 758; C. Gusy, Probleme der Verrechtlichung technischer Standards, NVwZ 1995, S. 105 ff., 107 ff.; Leisner (Fn. 23), S. 226 ff.; H. Hill, Normsetzung und andere Formen exekutivischer Selbstprogrammierung, in: W. Hoffmann-Riem/E. Schmidt-Aßmann/A. Voßkuhle (Hrsg.), Grundlagen des Verwaltungsrechts, Bd. 2, 2008, § 34, Rn. 45.

37 Vgl. im Zusammenhang des Polizeirechts etwa B. Pieroth/B. Schlink/M. Kniesel, Polizei- und Ordnungsrecht, 5. Aufl., 2008, S. 126 ff.; auch Ladeur (Fn. 7), S. 221. Diese Voraussetzung in Bezug auf die Funktion des parlamentarischen Gesetzes lässt sich auf die rechtsstaatliche Zielbestimmung zurückführen, die bestehenden Rechtszustände vor gesellschaftlichen Wandlungen zu bewahren. Zu diesem Aspekt des Rechtsstaates vgl. auch K. Hesse, Der Rechtsstaat im Verfassungssystem des Grundgesetzes, in: E. Forsthoff (Hrsg.), Rechtsstaatlichkeit und Sozialstaatlichkeit: Aufsätze und Essays, 1968, S. 557 ff., 571 f.; E. Forsthoff, Begriff und Wesen des sozialen Rechtsstaates, in: VVDStRL 12 (1954), S. 8 ff., 19; $B$. Schlink, Freiheit durch Eingriffsabwehr - Rekonstruktion der klassischen Grundrechtsfunktion, EuGRZ 1984, S. 457 ff., 463; H. Wissmann, Generalklauseln: Verwaltungsbefugnisse zwischen Gesetzmäßigkeit und offenen Normen, 2008, S. 127.

38 Der dynamische Charakter der Verwaltung hängt natürlich mit deren vielfältigen Funktionen und Aufgaben zusammen. Vgl. dazu nur G. F. Schuppert, Verwaltungswissenschaft: Verwaltung, Verwaltungsrecht, Verwaltungslehre, 2000, S. 73 ff. 
Rechtsbegriffe würde ohne weitere Konkretisierungen durch die Verwaltung die stabilisierende und steuernde Funktion des Gesetzes beeinträchtigen, so dass nicht (mehr) das Gesetz, sondern die (fachlich besonders kompetente) Verwaltung die maßstabbildende Aufgabe übernehmen müsse. ${ }^{39}$ Dabei wird indes vernachlässigt, dass selbst die im Umweltrecht viel verwendeten unbestimmten Rechtsbegriffe eine Anpassungsfähigkeit in sich tragen, da bereits dem Umweltrecht eine unter Ungewissheitsbedingungen erwachsende und daher stark dynamische Ordnungsidee zugrunde liegt. Anders als die klassischen unbestimmten Rechtsbegriffe, welche häufig eine inhaltsfeste Ordnungsidee zugrunde legen und zur Sicherstellung bestehender Rechtszustände im Rahmen dieser Ordnungsidee sogar eine (einzig) richtige Auslegung bzw. Anwendung voraussetzen, ${ }^{40}$ stützen sich die umweltrechtlichen unbestimmten Rechtsbegriffe angesichts der Dynamik und Ungewissheit der zu regelnden Materie eben nicht auf ein inhaltlich vorherbestimmbares Ordnungsdenken. Im Gegenteil setzen sie die stetigen Wandlungen des betreffenden Sachverhalts voraus und müssen deshalb durchaus dazu dienen, ein wandlungsfähiges Regelungsregime zu gewährleisten. Schon der offenbar zukunftsorientierte und ungewissheitsbezogene Charakter der den genannten unbestimmten Rechtsbegriffen zugrundeliegenden umweltrechtlichen Grundsätze, etwa des Vorsorge-, des Verursacher-, des Kooperations-, des Integrations- und des Nachhaltigkeitsprinzips ${ }^{41}$ bestätigt wohl diesen Befund. So gesehen verkennt die herrschende Meinung auch die bindende Bedeutung der umweltrechtlichen unbestimmten Rechtsbegriffe, indem sie diese lediglich als maßstablose und daher steuerungsunfähige offene Normen ansehen. Vor allem lässt sie außer acht, dass dem Umweltrecht eine wandlungsorientierte Ordnungsidee zugrunde liegt, so dass die umweltrechtlichen unbestimmten Rechtsbegriffe selbst die Verpflichtung der Verwaltung zur dynamischen und flexiblen Reaktion enthalten. Schon in dieser Hinsicht geht die allgemeine Einschätzung fehl, dass die unbestimmten Rechtsbegriffe allein nicht in der Lage seien, (gerichtliche) Kontrollmaßstäbe für die Verwaltungsentscheidung aufzustellen.

Dieser von der herrschenden Auffassung angenommene Ausgangspunkt führt zu der These, dass die Bindung einzelner Verwaltungsentscheidungen an das Gesetz sich vornehmlich nicht mehr am Maßstab der unbestimmten Rechtsbegriffe, sondern erst mit Hilfe der normkonkretisierenden Verwaltungsvorschriften verwirklichen lasse. Anders ausgedrückt tritt die normkonkretisierende Verwaltungsvorschrift an die Stelle des unbestimmten Rechtsbegriffs, indem sie die traditionell dem Gesetz zugehörigen Verantwortung übernimmt, möglichst vollständige Handlungsdirektiven für die Verwaltungs-

39 So deutlich bei Hill (Fn. 24), S. 407 f.; H. Kind, Ist die gesetzliche Verpflichtung der Exekutive zur (letztverbindlichen) Auslegung eines ,unbestimmten Rechtsbegriffes“ durch Allgemeine Verwaltungsvorschriften verfassungswidrig?, DÖV 1988, S. 679 ff., 680; M. Gerhardt, Normkonkretisierende Verwaltungsvorschriften, NJW 1989, S. 2233 ff., 2236 f.; SchulzeFielitz (Fn. 7), S. 779 f.

40 Zur These der einzig richtigen Entscheidung beim unbestimmten Rechtsbegriff vgl. schon Jesch (Fn. 6), S. 211 f., der davon ausging, bei der Ermessensentscheidung habe die Exekutive die Wahl zwischen mehreren Entscheidungen, von denen jede richtig sei, während es bei der Auslegung und Anwendung unbestimmten Rechtsbegriffs nur eine richtige Entscheidung geben könne. Diese Annahme findet auch heutzutage noch und immer wieder Zustimmung. Vgl. dazu nur Maurer (Fn. 10), Rn. $29 \mathrm{f}$.

41 Zur Zukunftsorientierung all dieser Grundsätze unter Ungewissheitsbedingungen vgl. nur $R$. Schmidt/W. Kahl, Umweltrecht, 7. Aufl., 2006, S. 8 ff.; Kloepfer (Fn. 8), S. 63 ff. 
entscheidung zur Verfügung zu stellen und dadurch zur Stabilisierung der Umweltrechtsordnung zu dienen. So betrachtet liegt es nahe, weshalb und wie die Verselbständigung der normkonkretisierenden Verwaltungsvorschriften von den (ermächtigenden) unbestimmten Rechtsbegriffen die Veränderung des Verhältnisses von Exekutive und Judikative herbeigeführt hat: Auf der einen Seite stellen die normkonkretisierenden Verwaltungsvorschriften eine verstärkte Bindungswirkung für die Gerichte dar, weil sie - genauso wie das Gesetz - für die Verwaltungsentscheidung maßgeblich sind. Auf der anderen Seite rechtfertigen sie sich allerdings eben dadurch, dass ihre Bindungsintensität stetigem Erkenntniswandel unterliegt. Demzufolge müsste die Verbindlichkeit der normkonkretisierenden Verwaltungsvorschriften gegenüber dem Gericht vor allem vom betreffenden Sachverstand abhängen. Das heißt, der Sachverstand der zur Standardisierung berufenen Behörde begründet und begrenzt zugleich die Bindungswirkung der normkonkretisierenden Verwaltungsvorschriften. ${ }^{42}$ Demgegenüber treten die unbestimmten Rechtsbegriffe im Rahmen der verwaltungsgerichtlichen Kontrolle ganz in den Hintergrund. Die allgemein anerkannten Überprüfungskriterien, etwa, ob die Standards willkürfrei ermittelt werden, ob sie hinreichend konservative Annahmen enthalten, ob sie nicht veraltet und nicht wegen Besonderheiten des Einzelfalles unanwendbar sind, haben daher mit den konkretisierten unbestimmten Rechtsbegriffen überhaupt nichts zu tun. Zusammenfassend lässt sich sagen, dass die Reduzierung bzw. Beschränkung gerichtlicher Kontrolldichte bei den normkonkretisierenden Verwaltungsvorschriften auf das (Miss)verständnis der umweltrechtlichen unbestimmten Rechtsbegriffe als maßstablose „Leer-Normen“ zurückzuführen ist, wobei die starke Abhängigkeit des Umweltrechts von technisch-wissenschaftlichen Erkenntnissen eine entscheidende Rolle spielt.

Vor dem dargelegten Hintergrund versteht sich, weswegen sich bei den normkonkretisierenden Verwaltungsvorschriften die gerichtliche Überprüfung nicht auf die unbestimmten Rechtsbegriffe, sondern auf den (dem Gericht nicht zugänglichen) technischwissenschaftlichen Sachverstand richtet: Da im Umweltrecht die unbestimmten Rechtsbegriffe nicht mehr mit der sog. einzig richtigen Antwort, sondern vielmehr mit der Einräumung behördlicher Beurteilungsspielräume verbunden seien, müsse die gerichtliche Kontrolle sich neben den allgemeingültigen rechtsstaatlichen Kriterien ${ }^{43}$ darauf beschränken und konzentrieren, ob die normkonkretisierenden Verwaltungsvorschrif-

42 So deutlich bei Wahl (Fn. 3), der einerseits annimmt, dass „Werte und Standards, die in normkonkretisierten Verwaltungsvorschriften enthalten sind, der gerichtlichen Kontrolle als Maßstäbe zugrundegelegt werden“ müssten, andererseits aber ausführt: „Normkonkretisierende Verwaltungsvorschriften können auch in ihrem inhaltlichen Anspruch auf Richtigkeit erschüttert werden, sie können veraltet und widerlegt sein. Eine Rechtsverordnung wäre im selben Fall bis zu ihrer formellen Abänderung gültig. Wegen dieser Bindungsschwäche verbleiben die normkonkretisierenden Verwaltungsvorschriften in einer Zwischenstellung zwischen den einfachen Verwaltungsvorschriften und der Rechtsverordnung. Die Gerichte prüfen bei ihnen in den Zweifel- und Sonderfällen mehr nach als bei den Rechtsverordnungen.“ Ähnlich vgl. auch Leisner (Fn. 23), S. 229.

43 Die rechtsstaatliche Kontrollfunktion des Gerichts betont $W a h l$ (Fn. 3), S. 412, 414. Zur Bedeutung der gerichtlichen Willkürlichkeitsprüfung im Rahmen einer allgemeinen Rechtmäßigkeitsprüfung vgl. auch $D$. Sellner, Atom- und Strahlenschutzrecht. Ein abgeschlossenes oder zukunftsweisendes Kapitel höchstrichterlicher Rechtsprechung?, in: FG BVerwG, 2003, S. $741 \mathrm{ff} ., 749 \mathrm{f}$. 
ten im Hinblick auf die aktuellen technisch-wissenschaftlichen Erkenntnisse anwendbar sind. ${ }^{44}$ Gerade diese Annahme läuft aber Gefahr, einen Raum für gerichtliche Willkür zu eröffnen, indem nicht mehr das Gesetz bzw. die unbestimmten Rechtsbegriffe, sondern vor allem die Sacherkenntnisse als Kontrollmaßstäbe im Mittelpunkt stehen. Wie dargelegt begründet die normkonkretisierende Verwaltungsvorschrift nicht unbedingt die Zurückhaltung der gerichtlichen Kontrolle, weil diese schon aus Gründen technischwissenschaftlicher Erkenntnisfortschritte von der normkonkretisierenden Verwaltungsvorschrift abweichen darf. Bereits die schwankende Stellung der normkonkretisierenden Verwaltungsvorschriften verdeutlicht, dass die Heranziehung des Sachverstandes nicht nur die bindende Funktion der unbestimmten Rechtsbegriffe für die Gesetzmäßigkeit der normkonkretisierenden Verwaltungsvorschriften beeinträchtigt, sondern dadurch auch die Gesetzesgebundenheit des Gerichts gefährdet. Aus dieser Perspektive ist festzustellen, dass die Begriffsentwicklung der normkonkretisierenden Verwaltungsvorschriften für die Verunsicherung des Verhältnisses nicht nur von Legislative und Exekutive, sondern von Exekutive und Judikative verantwortlich ist.

\section{Normkonkretisierende Verwaltungsvorschrift oder Rechtsverordnung?}

Um die Problematik der normkonkretisierenden Verwaltungsvorschriften hinsichtlich ihrer Bindungswirkung näher zu begreifen, ist eine Wiederentdeckung der Bedeutung der unbestimmten Rechtsbegriffe im Umweltrecht unentbehrlich. Wie angedeutet darf angesichts der Ungewissheit und Dynamik der zu regelnden Sachverhalte im Rahmen des Umweltrechts der unbestimmte Rechtsbegriff nicht für inhalts- und maßstablos gehalten werden. Der Verzicht des Gesetzgebers darauf, inhaltlich vorherbestimmte oder -bestimmbare Begriffe zu verwenden, bedeutet in dieser Hinsicht nicht, dass das Gesetz seine stabilisierende Funktion verloren hat, sondern vielmehr, dass das Gesetz selbst eine dynamisierende Funktion trägt. Das heißt, bereits die zahlreichen unbestimmten Rechtsbegriffe setzen die Wandelbarkeit und Temporärität der ihnen zugrundeliegenden Ordnungsidee voraus und besitzen daher keine hohe Konkretisierungsbedürftigkeit, sondern spiegeln die starke Anforderung an flexible Regelungsstrategien wider. ${ }^{45}$ So verstanden entfaltet weder die normkonkretisierende Verwaltungsvorschrift noch der ihr zugrunde liegende technisch-wissenschaftliche Sachverstand, sondern vielmehr der unbestimmte Rechtsbegriff die Bindungswirkung sowohl für die vollziehende als auch für die rechtsprechende Gewalt, indem dieser ein Ermächtigungselement enthält, welches zugleich auf die Verpflichtung der Verwaltung zu einer bestimmten Verhaltensweise verweist.

44 Dies ist weitgehend als „Rücknahme“ der gerichtlichen Überprüfung anerkannt. Vgl. etwa Schulze-Fielitz (Fn. 7), S. 779 f.; Hill (Fn. 24), S. 409.

45 Dies verdeutlicht sich schon bei der gesetzgeberischen Unterscheidung zwischen, ,allgemein anerkannten Regeln der Technik“, „Stand der Technik“ und „Stand von Wissenschaft und Technik", die abgestufte Anforderungen an die Verwaltung darstellt. Vgl. dazu etwa Hendler (Fn. 34), S. 482, 485; M. Schäfer, Verfassungsrechtliche Rahmenbedingungen für die Konkretisierung unbestimmter Sicherheitsstandards durch die Rezeption von Sachverstand, 1998, S. $29 \mathrm{ff}$. 
Mit Blick auf den Bindungsaspekt der umweltrechtlichen unbestimmten Rechtsbegriffe liegt es nahe, dass die enge Beziehung von den ermächtigenden unbestimmten Rechtsbegriffen und den sie konkretisierenden normkonkretisierenden Verwaltungsvorschriften weder aufgrund der Anerkennung behördlicher Beurteilungsspielräume noch durch die Hervorhebung der gesetzesgleichen Bedeutung von normkonkretisierenden Verwaltungsvorschriften auflösbar ist. Mit anderen Worten: Die normkonkretisierenden Verwaltungsvorschriften sind an die von ihnen konkretisierten unbestimmten Rechtsbegriffen zu binden. Da die unbestimmten Rechtsbegriffe als solche jedoch keine inhaltsfeste bzw. keine besonders dem Schutz bestehender Rechtszustände ${ }^{46}$ dienende Ordnungsidee voraussetzen, weisen sie auf keine inhaltsrichtigen Standards hin. Sie binden die normkonkretisierenden Verwaltungsvorschriften vielmehr dadurch, dass ihre Ermächtigung die zur Standardisierung berufene Behörde dazu verpflichtet, unter Ungewissheitsbedingungen reaktions- und aktualisierungsfähige Regelungsmaßnahmen zu unternehmen. Die gesetzliche Ermächtigung durch die unbestimmten Rechtsbegriffe bedeutet daher nicht pauschale Kompetenzübertragung auf die Exekutive, die infolgedessen jedenfalls intervenierende Regelungen vornehmen solle und dürfe. ${ }^{47}$ Sondern sie verweist mittels ihrer inhaltlichen Offenheit in erster Linie darauf, dass die exekutive Standardisierung sich nicht auf bestimmte technisch-wissenschaftliche Erkenntnisse beschränken darf und daher alle betreffenden Gesichtspunkte bzw. Interessen zu berücksichtigen hat. ${ }^{48}$ So gesehen setzen die beiden vielfach auftauchenden Begriffe „Stand von Wissenschaft und Technik ${ }^{449}$ und „schädliche Umwelteinwirkungen“50 als Konkretisierung- und Standardisierungsermächtigung einen Rahmen, innerhalb dessen

46 Zum Bestandsschutz im Rahmen des Umweltrechts vgl. etwa K. Hansmann, Der Bestandsschutz im Immissionsschutzrecht, in: FG BVerwG, 2003, S. 935 ff.

47 Bereits in dieser Hinsicht liegt es nahe, daß das umweltrechtliche Vorsorgeprinzip sich nicht immer auf die Risikominimierung, sondern vielmehr auf die vielfältige Interessenabwägung unter Risikobedingungen bezieht. Vgl. dazu auch H. Sendler, Normkonkretisierende Verwaltungsvorschriften im Umweltrecht, UPR 1993, S. 321 ff., 326. Für eine aktive Verwaltung unter Ungewissheitsbedingungen spricht aber etwa Ossenbühl (Fn. 36), S. 758; SchmidtPreuß (Fn. 3), S. 771. Demgegenüber macht Rüdiger Breuer darauf aufmerksam, dass die unbestimmten Rechtsbegriffe bzw. die „rechtlichen Lücken und Spielräume“ nicht immer für die behördlichen Beurteilungsspielräume, sondern ggf. für die Privatautonomie sprechen. Vgl. dazu Breuer (Fn. 3), S. 106.

48 Zur Aufgabe der Verwaltungsbehörde zur Interessenabwägung im Umwelt- und Technikrecht vgl. auch U. Di Fabio, Risikoentscheidungen im Rechtsstaat, 1994, S. 275, 286 ff.; K. Lange, Staatliche Steuerung durch offene Zielvorgabe im Lichte der Verfassung, VerwArch 82 (1991), S. 1 ff., 5 ff.; T. Groß, Die Rezeption von Erkenntnissen der Wissenschaft und Technik im Verwaltungsrecht, in: W. Gropp u. a. (Hrsg.), Rechtswissenschaft im Wandel, FS 400 Jahre Juristische Fakultät Gießen, 2007, S. 153 ff., 157; I. Appel, Methodik des Umgangs mit Ungewissheit, in: E. Schmidt-Aßmann/W. Hoffmann-Riem (Hrsg.), Methoden der Verwaltungsrechtswissenschaft, 2004, S. 327 ff., 336 ff.; I. Appel, Staatliche Zukunfts- und Entwicklungsvorsorge: Zum Wandel der Dogmatik des öffentlichen Rechts am Beispiel des Konzepts der nachhaltigen Entwicklung im Umweltrecht, 2005, S. 167, 169. Gleichwohl steht im Rahmen dieser Diskussionen häufig nicht die Bindungskraft des parlamentarischen Gesetzes bzw. des unbestimmten Rechtsbegriffs, sondern vielmehr der (zu erweiternde) Beurteilungsspielraum der Verwaltung im Vordergrund.

49 Dieser Begriff wird im Umwelt- und Technikrecht häufig gebraucht, exemplarisch dient $\S 7$ Nr. 3 AtomG.

50 Vgl. z. B. $\S 1, \S 5$ Nr. 1-2 BImSchG. 
die zuständige Behörde große inhaltliche Gestaltungsspielräume genießt. Dadurch wird dieser inhaltlich offene Rahmen aber nicht bindungsunfähig. Vielmehr liegt seine Grenze darin, dass die behördliche Konkretisierung bzw. Standardisierung nicht vom gesetzlichen Ermächtigungsgehalt, vor allem nicht vom Zweck der gesetzlichen Ermächtigung abweichen darf. Sowohl „Stand von Wissenschaft und Technik“ als auch „,schädliche Umwelteinwirkungen“ setzen im Umweltrecht unsicheres Wissen voraus ${ }^{51}$ und weisen insofern gemeinsam darauf hin, dass die behördliche Standardisierung die widerstreitenden technisch-wissenschaftlichen Gesichtspunkte bzw. Interessen umfassend abzuwägen hat, denn nur so lässt sich der Ermächtigungszweck erreichen, wonach die zuständige Behörde vornehmlich nicht dazu verpflichtet ist, die inhaltlich richtige Konkretisierung der genannten unbestimmten Rechtsbegriffe herauszufinden, sondern vielmehr dazu, die Dynamik und Ungewissheit der zu regelnden Materie in Betracht zu nehmen und gerade auf dieser Grundlage den widerstreitenden Interessen die Möglichkeit stetiger Auseinandersetzungen zu bewahren. Zum Beispiel stellt der Begriff „Stand von Wissenschaft und Technik“ im Atomgesetz im Vergleich zu den ,allgemein anerkannten Regeln der Technik“ einen höheren und stärker zukunftsbezogenen Standard auf, ${ }^{52}$ wonach die zuständige Behörde verpflichtet ist, sowohl technische als auch wissenschaftliche Gesichtspunkte in den Konkretisierungsprozess mit einzubeziehen, um einerseits dessen Wertoffenheit gegenüber technisch-wissenschaftlichen Debatten unter Ungewissheitsbedingungen darzustellen ${ }^{53}$ und andererseits gerade dadurch den speziellen Zweck des Atomrechts, Risiken der Kernenergie bestmöglich zu minimieren, ${ }^{54} \mathrm{zu}$ erreichen. Auch der Begriff „schädliche Umwelteinwirkungen“ im Rahmen des Bundesimmissionsschutzgesetzes bindet die Exekutive durch seine inhaltsoffene und doch aufgabenbestimmte Ermächtigung, wonach die Behörde bei der Bewertung von Umwelteinwirkungen alle vom Gesetz geschützten Interessen ${ }^{55} \mathrm{zu}$ berücksichtigen hat.

51 Vgl. auch A. Scherzberg, Wissen, Nichtwissen und Ungewissheit im Recht, in: C. Engel/J. Halfmann/M. Schulte (Hrsg.), Wissen - Nichtwissen - Unsicheres Wissen, 2002, S. 114 ff., 126-128; Appel, Staatliche Zukunfts- und Entwicklungsvorsorge (Fn. 48), S. 164.

52 Vgl. dazu z. B. R. Breuer, Gefahrenabwehr und Risikovorsorge im Atomrecht, DVBl. 1978, S. 829 ff., 837; P. Marburger, Atomrechtliche Schadenvorsorge. Möglichkeiten und Grenzen einer normativen Konkretisierung, 2. Aufl., 1985, S. 19 f.; Hendler (Fn. 34), S. 482, 485.

53 Vgl. in diesem Zusammenhang auch R. Breuer, Gefahrenabwehr und Risikovorsorge im Atomrecht, DVB1. 1978, S. 829 ff., 837 f.; S.-P. Hwang, Grundrechtsoptimierung durch (Kelsensche) Rahmenordnung: Zugleich ein Beitrag zur grundrechtsoptimierenden Funktion der unbestimmten Rechtsbegriffe am Beispiel „Stand von Wissenschaft und Technik“, Der Staat 49 (2010), S. 456 ff., 472 ff.

54 Zum Risikominimierungsgebot im Rahmen des Atomrechts vgl. Rehbinder (Fn. 17), S. 146 f.; D. Sellner/G. Hennenhöfer, Atom- und Strahlenschutzrecht, in: K. Hansmann/D. Sellner (Hrsg.), Grundzüge des Umweltrechts, 3. Aufl., 2007, S. 863 ff., 881 ff.; Kloepfer (Fn. 8), S. 240 .

55 Nach dem Zweck des Bundesimmissionsschutzgesetzes (vgl. nur § 1 BImSchG.) sind Menschen, Tiere und Pflanzen, der Boden, das Wasser, die Atmosphäre sowie Kultur- und sonstige Sachgüter vor schädlichen Umwelteinwirkungen zu schützen. Bereits diese Zweckbestimmung macht klar, dass im Rahmen dieses Gesetzes der Umweltschutz vielfältige Interessen umfasst, die in unterschiedlichen Entwicklungsphasen miteinander widerstreiten könnten und daher von Fall zu Fall auszugleichen sind. Vgl. zu diesem Aspekt des Bundesimmissionsschutzgesetzes und dessen Ermächtigung durch unbestimmte Rechtsbegriffe wie ,schädliche Umwelteinwirkungen“" auch Rehbinder (Fn. 17), S. 129. 
Kurz: Die Fragen, worauf sich der „Stand von Wissenschaft und Technik“ bezieht und welche Anlagen „schädliche Umwelteinwirkungen“ verursachen würden, lassen sich nach dem Ermächtigungsgehalt dieser Begriffe erst durch umfassende und dauerhafte Interessenauseinandersetzungen beantworten, deren Gewährleistung daher als Zentralaufgabe der Verwaltung zu bestimmen ist.

An dieser Stelle verdeutlicht sich, dass weder „Stand von Wissenschaft und Technik“ noch „schädliche Umwelteinwirkung" die Selbständigkeit der Verwaltung gegenüber dem Gesetz vorbehaltlos rechtfertigt. Vor allem dürfen diese unbestimmten Rechtsbegriffe nicht ausschließlich als Konkretisierung des mit der Entwicklung von der Gefahrenabwehr zur Risikovorsorge ${ }^{56}$ zunehmend verbreiteten umweltrechtlichen „Vorsorgeprinzips “57 missverstanden werden, dessen Geltendmachung mehr oder weniger einer Kompetenzerweiterung der Verwaltung bedürfte. ${ }^{58}$ Vielmehr ist darauf aufmerksam zu machen, dass das Nachhaltigkeitsprinzip, welches inzwischen als übergeordnetes Grundprinzip des Umweltrechts ${ }^{59}$ qualifiziert worden ist, eine ,integrative Beachtung ökonomischer, sozialer und ökologischer Entwicklungserfordernisse" verlangt. ${ }^{60}$ Das heißt, einer zukunftsorientierten nachhaltigen Entwicklung liegt nicht nur Risikominimierung, sondern auch Risikomanagement aufgrund Interessenausgleichung zugrunde. ${ }^{61}$ Denn: Ob eine Anlage umweltfreundlich oder -schädlich ist, lässt sich unter Ungewissheitsbedingungen nicht feststellen. ${ }^{62}$ Bereits dieser Aspekt weist darauf hin, dass angesichts der Dynamik und Ungewissheit zukünftiger Entwicklungen die umweltgesetzliche Ermächtigung durch die unbestimmten Rechtsbegriffe häufig keineswegs einseitig für die ausschließlich vorsorgeorientierte und insofern intensiv intervenierende staatliche Regelung zu sprechen vermag. Die inhaltliche Offenheit der Begriffe „Stand von Wissenschaft und Technik“ und „schädliche Umwelteinwirkungen“ setzt vielmehr die Entwicklungsdynamik und -ungewissheit voraus, die sich daher nicht durch inhaltliche Vervollständigung von normativen Vorgaben beseitigen, sondern nur durch viel-

$56 \mathrm{Zu}$ dieser Entwicklungstendenz im Umweltrecht vgl. nur Ladeur (Fn. 3), S. 69 ff.

57 Vgl. nur den Überblick bei Rehbinder (Fn. 17), S. 123 ff., 135 ff.

58 Zur schwerwiegenden Rolle der Verwaltung im Rahmen des Vorsorgeprinzips vgl. nur Appel, Staatliche Zukunfts- und Entwicklungsvorsorge (Fn. 48), S. 164 ff., $490 \mathrm{ff}$.

59 Zum Vorrang des Nachhaltigkeitsgrundsatzes vgl. W. Köck, Nachhaltigkeit im Verwaltungsrecht, Die Verwaltung 40 (2007), S. 419 ff., 432; W. Kahl, Der Nachhaltigkeitsgrundsatz im System der Prinzipien des Umweltrechts, in: H. Bauer/D. Czybulka/W. Kahl/A. Voßkuhle (Hrsg.), Umwelt, Wirtschaft und Recht, 2002, S. 111 ff., 138.

60 Zu dem sog. „drei-Säulen Konzept“ vgl. etwa Köck (Fn. 59), S. 424, 427; Appel, Staatliche Zukunfts- und Entwicklungsvorsorge (Fn. 48), S. 17, 35, 339 ff.; Rehbinder (Fn. 17), S. 159 ff.; E. Rehbinder, Nachhaltigkeit als Prinzip des Umweltrechts: konzeptionelle Fragen, in: K.-P. Dolde (Hrsg.), Umweltrecht im Wandel, 2001, S. 721 ff., 724 ff.

61 Auch in diesem Punkt verdeutlicht sich der Unterschied zwischen Vorsorge- und Nachhaltigkeitsprinzip. Vgl. dazu Kahl (Fn. 59), S. 138: „Während das Vorsorgeprinzip aber präventiv wirkt, indem es vorbeugen und das Entstehen von Umweltbelastungen unterhalb der Gefahrenschwelle vermeiden möchte, wirkt das Nachhaltigkeitsprinzip prospektiv und richtet den Umweltschutz auf die Zukunft aus im Sinne einer langfristigen-dauerhaften Ressourcensicherung." (Hervorhebung im Original)

62 Vgl. in diesem Zusammenhang auch Sellner (Fn. 43), S. 769: „Die Forderung nach der bestmöglichen Gefahrenabwehr und Risikovorsorge im Atomrecht korrespondiert mit dem für das gesamte technische Sicherheitsrecht wesentlichen Grundsatz, dass technische Betätigung dann nicht mehr möglich ist, wenn das Recht absolute Sicherheit fordert.“ 
fältige und stetige Interessenabwägung bewältigen läßt. So gesehen enthält die Ermächtigung der unbestimmten Rechtsbegriffe eine Verpflichtung der Verwaltung zur Abwägung nicht nur der bestehenden Rechtszustände, sondern auch aller angesichts der Eigenschaft des Umweltrechts in Betracht kommenden, zukunftsbezogenen und besonders im Rahmen des jeweiligen ermächtigenden Gesetzes eine Rolle spielenden Grundsätze.

Soweit die erwähnten umweltrechtlichen unbestimmten Rechtsbegriffe die Verwaltung nicht durch inhaltliche Richtigkeitshinweise, sondern durch zielbezogene Ermächtigung binden, sind sowohl die Verwaltung als auch die Gerichte nicht auf den Regelungsgehalt, sondern statt dessen auf den Ermächtigungsgehalt der unbestimmten Rechtsbegriffe auszurichten. Während ihr Regelungsgehalt nichts konkretes ausdrückt, legt ihr Ermächtigungsgehalt durchaus dar, weshalb eine Ermächtigung an die Verwaltung notwendig ist und wie diese die ermächtigten Aufgaben erfüllen soll. Wie gezeigt weist die inhaltliche Unbestimmtheit der genannten unbestimmten Rechtsbegriffe keineswegs auf deren Steuerungsunfähigkeit, sondern gerade auf die Notwendigkeit umfassender Berücksichtigung von unterschiedlichen Interessen hin, die angesichts der starken Zukunftsorientierung des Umweltrechts über die Gewährleistung bestehender Rechtszustände hinausgeht. So verstanden liegt es nahe, dass die Verselbständigung der normkonkretisierenden Verwaltungsvorschriften von den ermächtigenden unbestimmten Rechtsbegriffen im Rahmen der Verwaltungsgerichtsbarkeit zur Verkennung der gerichtlichen Kontrollmaßstäbe führen würde, indem nicht der unbestimmte Rechtsbegriff bzw. sein Ermächtigungsgehalt, sondern der technisch-wissenschaftlicher Sachverstand im Mittelpunkt steht. Konzentriert sich das Gericht ausschließlich darauf, ob die Standards willkürfrei ermittelt sind, ob sie hinreichend konservative Annahmen enthalten, ob sie veraltet oder wegen Besonderheiten des Einzelfalles unanwendbar sind, so lässt das Gericht die lenkende Funktion der unbestimmten Rechtsbegriffe außer Acht, da nach allgemeiner Ansicht die genannten Kriterien für die gerichtliche Kontrolle nicht auf die Ermittlung des Ermächtigungsgehaltes der unbestimmten Rechtsbegriffe zurückgehen müssten. Besonders kommt bei der gerichtlichen Überprüfung kaum in Betracht, ob die in Rede stehende normkonkretisierende Verwaltungsvorschrift die ermächtigte Aufgabe erfüllt hat, die widerstreitenden Gesichtspunkte und Interessen entsprechend dem Zweck und innerhalb der Grenzen der jeweiligen gesetzlichen Ermächtigung miteinander abzuwägen. ${ }^{63}$ Dies hat zur Folge, dass die angebliche Bindungswirkung der normkonkretisierenden Verwaltungsvorschriften, die von der Aktualität der behördlichen und sogar der gerichtlichen Erkenntnisse abhängen müsste, wegen der Dynamik und Ungewissheit des technisch-wissenschaftlichen Erkenntnisstandes auf der einen Seite und ohne normativ stabile Kontrollmaßstäbe auf der anderen Seite auch ungesichert bleibt. Im Ergebnis kann trotz und auch wegen Anerkennung der (bedingten) Bindungswirkung normkonkretisierender Verwaltungsvorschriften weder die gerichtliche Kompetenzüberschreitung noch die behördliche Willkür bei der Standardsetzung verhindert werden.

63 So gesehen fehlt bei der gerichtlichen Überprüfung normkonkretisierender Verwaltungsvorschrift gerade ein normativ verankerter Kontrollmaßstab, wie dies bei der Gerichtskontrolle von Ermessensentscheidung ( $\$ 40 \mathrm{VwVfG}$ und $\S 114 \mathrm{VwGO}$ ) der Fall ist. 
All dies ist darauf zurückzuführen, dass weder die behördliche Standardisierung noch deren gerichtliche Überprüfung die Bindungswirkung der unbestimmten Rechtsbegriffe ernst nimmt. Dient nicht der Ermächtigungsgehalt der unbestimmten Rechtsbegriffe, sondern der technisch-wissenschaftliche Sachverstand als Prüfungsmaßstab für die Richtigkeitsgewähr der normkonkretisierenden Verwaltungsvorschriften, so ergibt sich zwangsläufig, dass weder die Verwaltung noch das Gericht nach Maßgabe der Ermächtigung der unbestimmten Rechtsbegriffe handeln müsste. Dabei wird allerdings übersehen, dass die fachliche Legitimation der normkonkretisierenden Verwaltungsvorschriften deren Rechtmäßigkeit bzw. Rechtsgebundenheit nicht begründen kann. Soweit die normkonkretisierenden Verwaltungsvorschriften unmittelbar nach außen wirken und insofern Verbindlichkeit auch gegenüber dem Gericht entfalten, unterliegen sie, genauso wie die Rechtsverordnungen, der Rechtmäßigkeitskontrolle vor allem am Maßstab des Ermächtigungsgehaltes der unbestimmten Rechtsbegriffe, wobei besonders zu prüfen ist, ob und wie die im Wege der Standardisierung widerstreitenden Interessen oder Gesichtspunkte umfassend abgewogen worden sind. So gesehen dürften die Rechtsverordnungen, deren Bindung an das Gesetz bzw. die ermächtigenden unbestimmten Rechtsbegriffe durch die stark an der gesetzlichen Ermächtigung orientierten gerichtlichen Überprüfung gesichert wird, eine bessere Handlungsform darstellen als die normkonkretisierenden Verwaltungsvorschriften, denn sie finden ihre verfassungsrechtliche Grundlage im Art. 80 Abs. 1 GG und gewährleisten damit den Vorrang des Gesetzgebers vor der Verwaltung im Rahmen der allgemeinen Rechtsetzung. ${ }^{64}$ Demgegenüber würden die nach außen wirkenden und ggf. bindenden normkonkretisierenden Verwaltungsvorschriften die kompetenzverteilende Funktion des Art. 80 Abs. 1 GG beeinträchtigen, ${ }^{65}$ indem sie aus sog. funktionell-rechtlichen Überlegungen entweder als Rechtsetzung innerhalb des behördlichen Beurteilungsspielraums oder sogar gewissermaßen als Gesetzgebung kategorisiert werden. Abgesehen von der debattierten Frage, inwiefern Art. 80 Abs. 1 GG die Rechtsetzungsprärogative des Parlaments begründet und damit die eigenständige exekutive Rechtsetzungsbefugnis ausschließt, ${ }^{66}$ hält diese Vorschrift nach herrschender Meinung durchaus an der Wesentlichkeitstheorie fest, ${ }^{67}$ wonach in allen „wesentlichen“ Bereichen die (nach der Wesentlichkeitsdoktrin noch

64 Vgl. dazu nur Hill (Fn. 36), Rn. 18 f.

65 Vgl. auch Wolf(Fn. 32), S. 856; Breuer (Fn. 3), S. 112; Saurer (Fn. 18), S. 265; J. Saurer, Die Funktionen der Rechtsverordnung: Der gesetzgeberische Zuschnitt des Aufgaben- und Leistungsprofils exekutiver Rechtsetzung als Problem des Verfassungsrechts, ausgehend vom Referenzgebiet des Umweltrechts, 2005, S. $343 \mathrm{ff}$.

66 Wie bereits angedeutet, handelt es sich hier um die Frage, ob Art. 80 Abs. 1 GG nur dem Vorbehaltsbereich der Legislative zugehört oder gar keine originäre Rechtsetzungsbefugnis der Exekutive zulässt. An einem gesetzlichen „Totalvorbehalt“ für den Verordnungserlass im Rahmen des Art. 80 Abs. 1 GG orientiert sich etwa Saurer (Fn. 18), S. 266 f. („Der in Art. 80 Abs. 1 GG statuierte Gesetzesvorbehalt sieht für den Verordnungserlass nach seinem Wortlaut keine Ausnahmen vor. Auch aus der systematischen Stellung des Art. 80 Abs. 1 GG lässt sich nichts anderes herleiten.") Vorsichtig auch M. Brenner, in: v. Mangoldt/Klein/Starck, Das Bonner Grundgesetz: Kommentar, 4. Aufl., 2001, Bd. 3, Art. 80, Rn. 7 ff., 24 ff.; Jachmann (Fn. 23), S. 23.

67 Vgl. nur E. Schmidt-Aßmann, Die Rechtsverordnung in ihrem Verhältnis zu Gesetz und Verwaltungsvorschrift, in: P. Kirchhof/M. Lehner/A. Raupach/M. Rodi (Hrsg.), Staaten und Steuern. Festschrift für Klaus Vogel zum 70. Geburtstag, 2000, S. 477 ff., 479 ff. 
zulässige) exekutive Rechtsetzungskompetenz sich erst vom Gesetz ableiten lässt und daher die Rückbindung an das Gesetz darstellen muss. Soweit die normkonkretisierenden Verwaltungsvorschriften die allgemeine Konkretisierungs- und Standardisierungsaufgabe tragen, reichen sie offenbar über die „unwesentlichen“ Entscheidungen hinaus, indem sie gesetzgeberische Funktion besitzen und gewissermaßen als Gesetze fungieren. Schon in dieser Hinsicht ist es fraglich, ob die normkonkretisierenden Verwaltungsvorschriften sich tatsächlich auf die exekutive originäre Rechtsetzungsbefugnis stützen und sich dergestalt der normativen Kontrolle nach Art. 80 Abs. 1 GG entziehen dürften. Auch die Rechtfertigung der normkonkretisierenden Verwaltungsvorschriften durch die normative Ermächtigungslehre, die auf den ersten Blick an der Verbindung des exekutiven Verordnungsrechts mit der gesetzlichen Ermächtigung festhält, läuft Gefahr, die kompetenzabgrenzende Bedeutung des Art. 80 Abs. 1 GG zu verzerren, da auch die normative Ermächtigungslehre den im Art. 80 Abs. 1 GG verkörperten Bindungsaspekt der Gesetzesermächtigung verkennt und daher die normkonkretisierenden Verwaltungsvorschriften nicht vor die Grenze gesetzlicher Ermächtigung stellt, sondern Beurteilungsspielräume eröffnet.

Im Hinblick auf die Regelung des Art. 80 Abs. 1 GG verdeutlicht sich also, dass die normkonkretisierenden Verwaltungsvorschriften die verfassungsrechtlichen Grenzen exekutiver Rechtsetzung überschreiten würden. Auf der Grundlage einer parlamentarischen Demokratie sieht Art. 80 Abs. 1 GG ausdrücklich vor, dass die Gesetzesbindung erst nach Maßgabe der Gesetzesermächtigung zu verwirklichen ist. Demzufolge darf auch der besondere Sachverstand der Verwaltung nicht von den inhaltsoffenen und doch richtungsbestimmten Direktiven der ermächtigenden unbestimmten Rechtsbegriffe abweichen. Übernimmt die Exekutive die allgemeine Rechtsetzungsaufgabe nicht durch Rechtsverordnungen, sondern mittels der normkonkretisierenden Verwaltungsvorschriften, die einerseits Außen- und Bindungswirkung entfalten, andererseits aber nicht von Art. 80 Abs. 1 GG geregelt werden müssten, so ist eine verfassungsrechtlich bedenkliche Kompetenzausdehnung der Verwaltung nicht zu vermeiden. So betrachtet erregen die normkonkretisierenden Verwaltungsvorschriften, die ggf. auch „wesentliche" bzw. „gesetzvertretende“ Entscheidungen treffen, sowohl verfassungsrechtliche als auch verwaltungsrechtliche Zweifel, während Rechtsverordnungen die Rückbindung an die gesetzliche Ermächtigung gewährleisten müssen und dergestalt dazu beitragen, die Gesetzmäßigkeit der Verwaltung zu verankern.

\section{Resümee}

Fassen wir zusammen: Die Begriffsentwicklung der normkonkretisierenden Verwaltungsvorschriften im Umweltrecht, die die Außen- und Bindungswirkung der exekutiven Rechtsetzung entweder durch die Einräumung behördlicher Beurteilungsspielräume oder durch die Begründung des originären Verordnungsrechts der Verwaltung zu entfalten versucht, führt zur Verselbständigung der behördlichen Konkretisierung bzw. Standardisierung von den konkretisierten unbestimmten Rechtsbegriffen und damit von der gesetzlichen Ermächtigung. Wie dargelegt löst diese Verselbständigung die Gesetzesbindung auf, indem sowohl die Verwaltung als auch das Gericht die Inhaltslosigkeit der unbestimmten Rechtsbegriffe voaussetzen und unter dieser Voraussetzung die Not- 
wendigkeit der technisch-wissenschaftlichen Erkenntnisse für die behördliche Maßstabbildung hervorheben, die daher die Unbestimmtheit der unbestimmten Rechtsbegriffe retten und diese insofern ersetzen sollen. Im Hinblick darauf, dass gerade die inhaltliche Offenheit der unbestimmten Rechtsbegriffe, welche sich aus der hohen Dynamik und erheblichen Ungewissheit der Entwicklungen im Umweltrecht ergibt, auf die Verpflichtung der (behördlichen sowie gerichtlichen) Rechtsanwendung zur umfassenden Berücksichtigung und Abwägung aller betreffenden widerstreitenden Interessen hinweist, ist jedoch festzustellen, dass der erwähnte Entwicklungsansatz, der auf dem Missverständnis der bindenden Bedeutung der unbestimmten Rechtsbegriffe beruht, die Gesetzesherrschaft und -bindung im Gefüge der Staatsfunktionen gefährden würde. Dürfte die normkonkretisierende Verwaltungsvorschrift die Rechtsverordnung funktional ersetzen, so würde Art. 80 Abs. 1 GG seine Sperrwirkung verlieren. Dabei ist nicht nur zu befürchten, dass an die Stelle der demokratischen Willensbildung im Rahmen der Gesetzgebung der besondere Sachverstand der Verwaltung treten würde. Zweifelhaft ist ferner, ob die Herausstellung der technisch-wissenschaftlichen Erkenntnisse, die auf den ersten Blick die zentrale Rolle für die Auffassung des geregelten Sachverhalts im Umweltrecht spielen, in der Tat nicht zur Vernachlässigung der Eigenschaft umweltrechtlicher Ordnungsideen führen würde. Soweit die behördliche Standardsetzung einen wertenden Charakter besitzt, unterliegt sie der gesetzlichen und daher der gerichtlichen Kontrolle. Das heißt, der Ermächtigungsgehalt des Gesetzes bzw. der unbestimmten Rechtsbegriffe setzt einen Rahmen, innerhalb dessen die behördliche Konkretisierung und Standardisierung die Rückbindung an die jeweiligen gesetzlichen Ordnungsgedanken darzustellen haben, die sich häufig nicht (lediglich) auf den Schutz bestehender Rechtszustände, sondern vor allem auf eine nachhaltige Zukunftsentwicklung beziehen. Die normative Anforderung an die Verwaltung, die betreffenden Sachverhalte willkürfrei und zwar aufgrund umfassender Interessenabwägung zu ermitteln und zu bewerten, ist daher nicht bloß an rechtsstaatlich allgemeingültige Grundsätze, sondern vielmehr an Inhalt, Zweck und Ausmaß individueller gesetzlicher Ermächtigungen anzuknüpfen. 